\title{
Safety and usefulness of needle-guided resection of levator muscles in laparoscopic abdominoperineal resection for low rectal cancer
}

\author{
Takehito Yamamoto, Akiyoshi Kanazawa, Hiroyuki Matsubara, Takuya Okamoto \\ Department of Gastroenterological Surgery and Oncology, Kitano Hospital, The Tazuke Kofukai Medical Research Institute, Osaka, Japan
}

Videosurgery Miniinv 2016; 11 (3): 186-191 DOI: $10.5114 /$ wiitm.2016.61386

\begin{abstract}
Introduction: During laparoscopic abdominoperineal resection (APR) for low rectal cancer, it is difficult to resect the levator muscles and remove a cylindrical specimen without venturing close to the rectal wall to ensure negative circumferential resection margins (CRM). To solve this problem, we developed a needle-guided, laparoscopic, abdominoperineal resection ( $L A P R)$ technique.

Aim: To present the safety and superiority of our technique, "needle-guided LAPR".

Material and methods: In 2015, we performed needle-guided LAPR in 5 patients. In brief, the procedure is performed as follows. After total mesorectum excision to the level of the levator muscles, a needle is inserted through the perineum from the dorsal side of the internal aspect of the anus toward the sacral tip. The levator muscles and fat tissue are resected laparoscopically by following the needle. After the levator muscles have been resected, the needle is followed in a similar manner to resect the specimen from the perineum, enabling easy access to the intra-abdominal space and removal of the specimen. No position change is required during the perineal operation or pelvic floor reconstruction.

Results: Mean age was 68 years and 3 patients were male. There were no intraoperative complications or conversions to open surgery. The mean operation time and intraoperative blood loss were 319 min and $131 \mathrm{ml}$, respectively. All specimens were cylindrical in shape and had negative CRM. There were no postoperative complications.

Conclusions: Needle-guided LAPR was easily and safely performed to achieve accurate resection of the levator muscles. This technique could contribute to standardization of LAPR.
\end{abstract}

Key words: abdominoperineal resection, circumferential resection margin, rectal cancer, laparoscopic abdominoperineal resection.

\section{Introduction}

During laparoscopic abdominoperineal resection (APR) for low rectal cancer, it is important to ensure negative circumferential resection margins (CRM). Laparoscopic APR is superior to open APR in that it enables precise visualization of the small intra-pelvic space, making it possible to accurately dissect the mesorectum and prevent injury to the ureter and autonomic nerves. Moreover, an operator and an assistant or a trainee can share the same view of the deep intra-pelvic space, which is an advantage in terms of education and standardization of surgical techniques. However, even with laparoscopic surgery it is still difficult to accurately resect the levator muscles while ensuring negative CRM. The specimen can be resected from the prostate and seminal vesicle in men or vaginal wall in women on the ventral side;

\section{Address for correspondence}

Dr. Takehito Yamamoto, Department of Gastroenterological Surgery and Oncology, Kitano Hospital, The Tazuke Kofukai Medical Research Institute, 2-4-20 Ogimachi, Kita-ku, Osaka 530-8480, Japan, phone: +81 08031176656, e-mail: tkht26@me.com 
however, on the dorsal side of the rectum there are no visible landmarks to follow when resecting the levator muscles. This explains why surgeons usually venture close to the rectal wall, creating a "surgical waist" in the specimen or causing rectal perforation or both. Indeed, the rate of CRM involvement, and therefore the rate of local recurrence, is reportedly higher for APR than for anterior resection (AR) [1-4]. To address these problems with APR, Holm et al. reported extralevator abdominoperineal resection (ELAPR, cylindrical APR) in 2007 [5]. In this procedure, extended perineal dissection, including the sphincter complex, is performed and the inferior surface of the levator muscles followed laterally to their point of origin on the pelvic sidewall. This procedure does make it possible to create a cylindrical specimen and ensure negative CRM. However, it necessitates moving the patient into the prone jackknife position and reconstruction of the pelvic floor, which can lead to an increase in operation time and intraoperative blood loss. We therefore developed the "needle-guided, laparoscopic, abdominoperineal resection (LAPR)" technique, which enables even relatively inexperienced surgeons to easily, safely, and accurately resect the levator muscles and create a cylindrically shaped specimen with negative CRM simply by inserting a needle from the perineum; no intraoperative position change or pelvic floor reconstruction is required.

\section{Aim}

The aim of our study is to present the safety and superiority of our technique, "needle-guided LAPR", to the conventional method for low rectal cancer.

\section{Material and methods}

\section{Patients}

We first implemented needle-guided LAPR in 2015. This method is applicable to patients with low rectal cancer $(\mathrm{Rb})$ located within $3 \mathrm{~cm}$ of the anal verge who require APR to enable confirmation of the distal surgical margin. Patients with local invasion of the levator muscle or other organs are not suitable candidates for this procedure. All procedures were performed by the same experienced laparoscopic colorectal surgeon, who is certified by the Japanese Society for Endoscopic Surgery. The study protocol was approved by the Institutional Review Board of Kitano Hospital, the Tazuke Kofukai Medical Research Institute.

\section{Surgical technique: part 1, total mesorectal} excision (TME)

The patient is placed in a lithotomy Trendelenburg position, and this position is maintained throughout the entire procedure. The operator stands on the patient's right side, the first assistant on the left, and the camera holder cranially on the patient's right side. A monitor is placed by the patient's left foot. A pneumoperitoneum is then created with a pressure of $10 \mathrm{~mm} \mathrm{Hg}$ and trocars placed as shown in Figure 1. Trocars $B$ and $D$ are placed lateral to the inferior epigastric artery and vein midway between the umbilicus and upper edge of the pubis. Trocars $A$ and $C$ are placed at the distance of a fist from trocars $B$ and $D$, respectively. Trocars $A$ and $D$ are $5 \mathrm{~mm}$, and trocars $B, C$, and $E$ are $12 \mathrm{~mm}$. Trocars $A$ and $B$ are used by the operator and trocars $C$ and $D$ by the first assistant. The camera is inserted through trocar $\mathrm{E}$ at the umbilicus, and its position remains unchanged throughout the operation. As in other laparoscopic surgery procedures for low rectal cancer, the sigmoid colon and rectum are completely mobilized, the inferior mesenteric artery (IMA) cut at its root, and the adjacent lymph nodes resected. The total mesorectal excision (TME) is then performed to

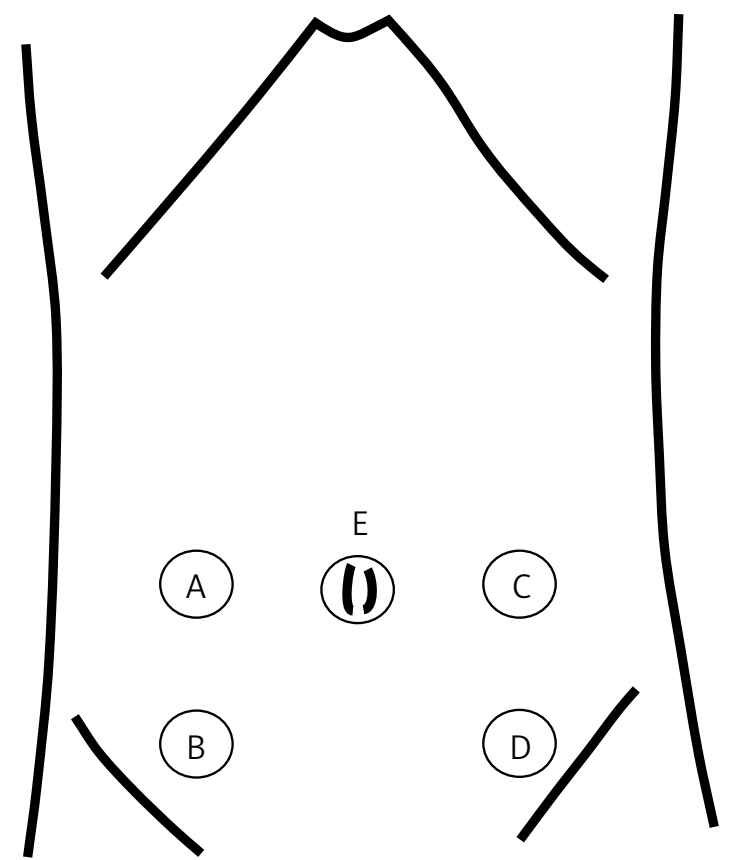

Figure 1. Trocar placement for laparoscopic colorectal surgery (trocars A and D: $5 \mathrm{~mm}$, trocars $B$ and $C: 12 \mathrm{~mm}$, trocar E: $12 \mathrm{~mm}$ ) 


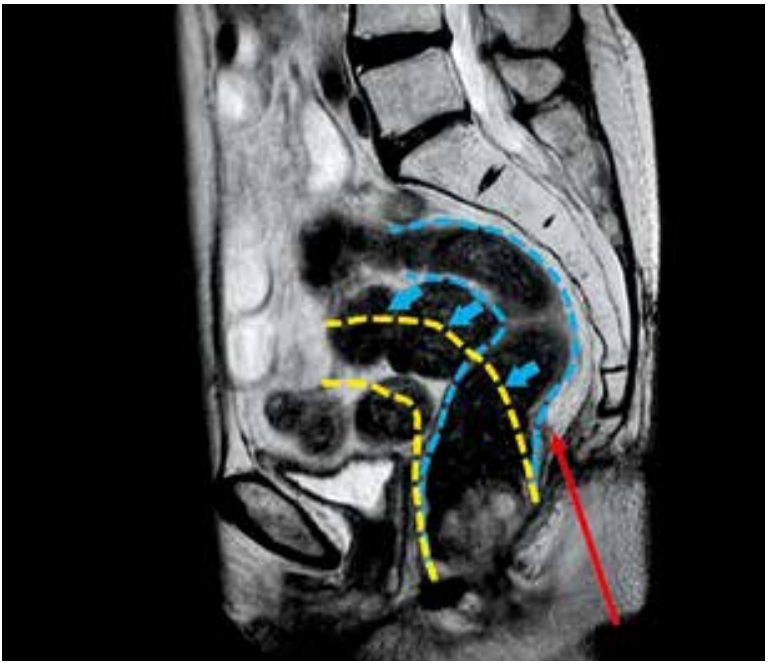

Photo 1. Magnetic resonance imaging image of patient 5 and schema showing the direction of insertion of the needle

the level of the surfaces of the levator muscles bilaterally on the dorsal side and to the caudal tip of the prostate in men or about the same level of the vaginal wall in women on the ventral side. The ureter and pelvic autonomic nerves are carefully protected during dissection. The sigmoid colon is severed with a linear stapler. The TME is not continued between the mesorectum and levator muscles. Instead, the levator muscles are dissected vertically distally to create a cylindrical specimen.

\section{Surgical technique: part 2, needle-guided resection of the levator muscles}

Next, the surgeon moves to the perineum, makes a skin incision that encircles the anus, inserts a needle anterior to the sacrum from the tip of the coccyx (Photos 1,2 ), and then moves back to the right side of the

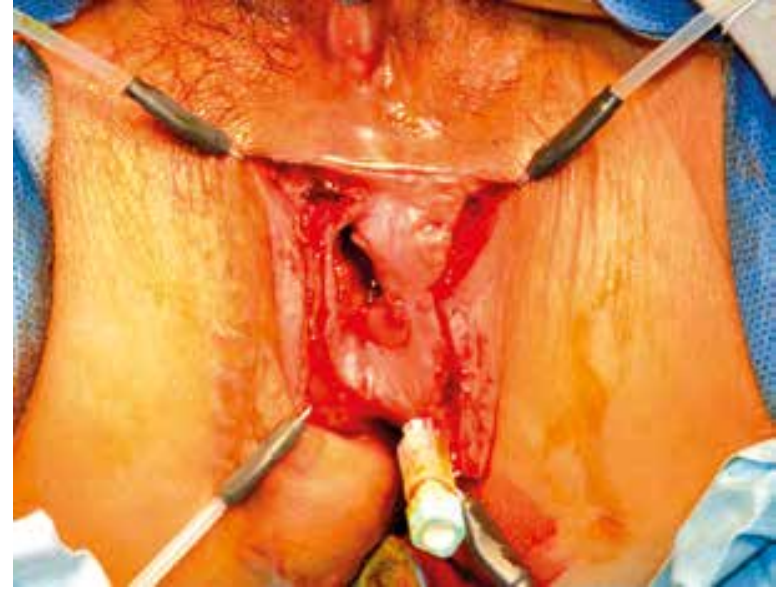

Photo 2. Inserted needle viewed from the perineum

patient. The levator muscles and fat tissue are resected laparoscopically following the needle, which emerges from the levator muscles (Photo 3). In this step, the first assistant uses forceps to elevate the mesorectum enough to enable clear visualization of the pelvic floor. After the levator muscles have been resected, the surgeon again moves to the perineum and resects the specimen from the perineum by similarly following the needle, this procedure enabling easy access to the intra-abdominal space. The specimen is then removed and a $19 \mathrm{Fr}$ double cannula drain inserted into the pelvic space from the wound of trocar D. Finally, the abdominal and perineal incisions are closed and a sigmoid stoma created on the left side of the abdomen.

\section{Results}

Laparoscopic, abdominoperineal resection was performed on 26 patients in our institution from 2009 to 2015, the first 21 of whom underwent stan-
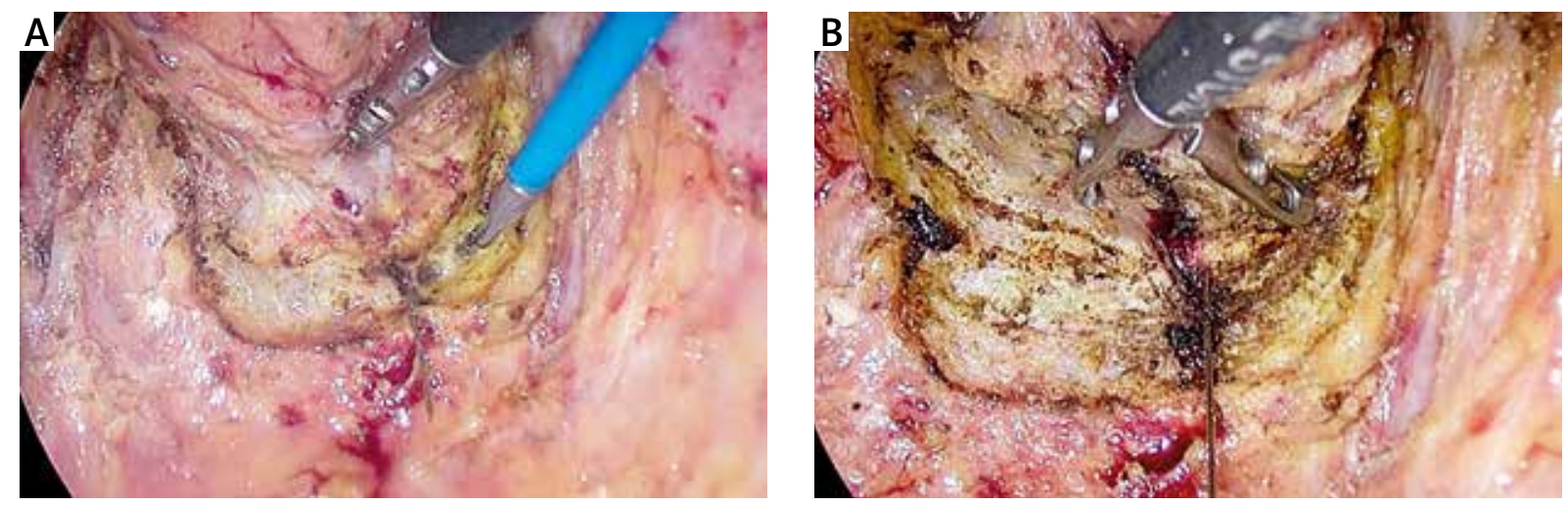

Photo 3. Laparoscopic view of the inserted needle emerging from the levator muscles 
dard LAPR. In 2015, needle-guided LAPR was developed and performed on the last five of these patients. Their mean age was 68 years and three were male. Relevant patient characteristics are shown in Table I. Patients 1 and 4 underwent neoadjuvant chemo-radiation therapy. No patient had levator muscle invasion or lateral lymph node metastases.

The operative and pathological findings are shown in Table II. No rectal perforations occurred and no patient required conversion to open surgery. The mean operation time was 319 min and intraoperative blood loss $131 \mathrm{ml}$. All specimens had negative CRM. A representative resected specimen (patient 5) is shown in Photo 4. The mean time to extracting the drain was 5 days, and the mean postoperative hospital stay was 15 days. There were no surgical site infections and no other postoperative complications (Clavien-Dindo classification > II). No patient developed obvious urinary or erectile dysfunction.

\section{Discussion}

The rate of recurrence is reportedly higher after APR than AR, and the prognosis is poorer [1-4]. This difference is attributable to the difficulty of resecting the levator muscles without venturing too close to the rectal wall $[6,7]$, which carries a risk of positive CRM and intraoperative rectal perforation, especially in obese patients with narrow pelvic spaces. Recently, many authors have reported the effectiveness of extralevator abdominoperineal resection (ELAPR, cylindrical APR) [5-9]. In this procedure, first reported by Holm et al., the levator muscles are totally resected from the pelvic wall, thus creating a cylindrical specimen and minimizing positive CRM. In a multicenter study, West et al. analyzed the pathological findings in 176 specimens from patients who had undergone ELAPR and reported that this procedure is associated with a lower incidence of CRM involvement and intraoperative rectal perforation than standard APR [6]. Similarly, Han et al. conducted a randomized controlled trial to compare ELAPR with conventional APR and found by pathological examination of surgical specimens that ELAPR achieves a larger total cross-sectional tissue area around the tumor than conventional APR [10]. Additionally, Chi et al. reported that ELAPR can be safely performed laparoscopically without position change. However, ELAPR is associated with high rates of perineal pain and pelvic infection because of the large dead space in the pelvic floor. Reconstruction of the pelvic defect with the assistance of plastic surgeons is frequently

Table I. Preoperative patient characteristics

\begin{tabular}{|c|c|c|c|c|c|c|c|c|c|}
\hline Patient & Gender & $\begin{array}{c}\text { Age } \\
\text { [years] }\end{array}$ & nCRT & $\begin{array}{c}\text { Stage } \\
\text { before } n C R T\end{array}$ & $\begin{array}{c}\text { Stage before } \\
\text { surgery }\end{array}$ & Height $[\mathrm{cm}]$ & $\begin{array}{c}\text { Body } \\
\text { weight }[\mathrm{kg}]\end{array}$ & $\mathrm{BMI}\left[\mathrm{kg} / \mathrm{m}^{2}\right]$ & $\begin{array}{l}\text { ASA } \\
\text { class }\end{array}$ \\
\hline 1 & Male & 67 & Yes & cT3,N1,M0 & yсT3,NO,MO & 168 & 65.7 & 23.3 & 2 \\
\hline 2 & Female & 63 & No & - & cT1b,NO,MO & 157.5 & 41.8 & 16.9 & 2 \\
\hline 3 & Male & 83 & No & - & cT3,NO,MO & 142 & 49.7 & 24.6 & 2 \\
\hline 4 & Male & 50 & Yes & cT3,NO,MO & cT3,NO,MO & 166.7 & 69.9 & 25.2 & 2 \\
\hline 5 & Female & 78 & No & - & cT3,NO,MO & 149.4 & 42.6 & 19.1 & 2 \\
\hline
\end{tabular}

$B M I$ - body mass index, ASA - American Society of Anesthesiologists, nCRT - neoadjuvant chemo-radiation therapy.

Table II. Operative and pathological characteristics of study procedures

\begin{tabular}{|lccccccc|}
\hline Patient & $\begin{array}{c}\text { Operation time } \\
{[\mathrm{min}]}\end{array}$ & $\begin{array}{c}\text { Blood loss } \\
{[\mathrm{ml}]}\end{array}$ & Complications & $\begin{array}{c}\text { Hospital stay } \\
{[\text { day }]}\end{array}$ & $\begin{array}{c}\text { Time to drain } \\
\text { removal [day] }\end{array}$ & $\begin{array}{c}\text { Pathological } \\
\text { stage }\end{array}$ & CRM \\
\hline 1 & 323 & 137 & No & 13 & 6 & ypT3,NO,MO & Negative \\
\hline 2 & 232 & 105 & No & 19 & 5 & pT2,NO,MO & Negative \\
\hline 3 & 368 & 40 & No & 16 & 3 & pT3,NO,MO & Negative \\
\hline 4 & 366 & 287 & No & 10 & 8 & ypT3,NO,MO & Negative \\
\hline 5 & 306 & 87 & No & 17 & 5 & pT3,NO,MO & Negative \\
\hline
\end{tabular}

CRM - circumferential resection margin 


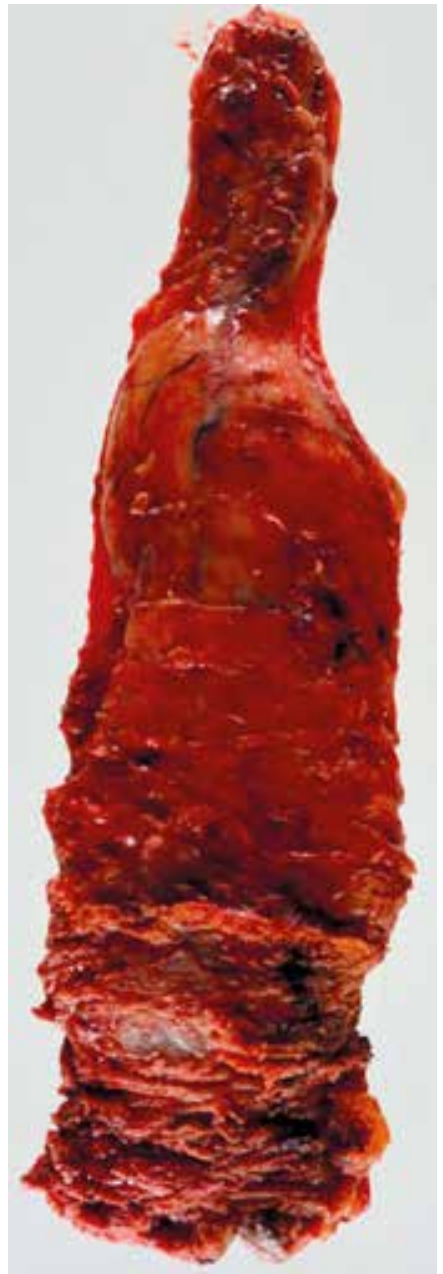

Photo 4. Operative specimen of patient 5. The specimen is cylindrically shaped, without a surgical waist

necessary, which prolongs the operation time. Moreover, total resection of the levator muscles is often not indicated from an oncological perspective and leads to unnecessarily invasive surgery with coccygeal resection in many patients [6, 10-12]. In addition, Prytz et al. reported that there is no difference between ELAPR and conventional APR in the rate of local recurrence and 3-year survival [13].

We believe that needle-guided LAPR has the following three advantages. First, it enables even relatively inexperienced surgeons to easily and safely resect the levator muscles and create a cylindrical specimen without venturing too close to the rectal wall. Second, it is easy to accurately resect the levator muscles laparoscopically under very clear visualization without creating a defect that requires pelvic defect reconstruction, which means shorter operation time and less intraoperative blood loss. Third, this technique enables experienced surgeons and trainees to share the same view of the deep pelvic space, which contributes to better education and standardization of the procedure.

This procedure has some limitations. It is contraindicated in patients with tumors that have extensively invaded the levator muscles, because of the risk of inserting the needle into the tumor and subsequent implantation. The feasibility of this procedure must be carefully examined preoperatively by preoperative computed tomography and magnetic resonance imaging scans. We have performed too few of these procedures to draw accurate conclusions about safety and oncological effectiveness. We need to perform the procedure on more patients and more precisely analyze specimens and outcomes to assess local recurrence rates and survival.

\section{Conclusions}

Needle-guided resection of levator muscles in LAPR could be easily and safely performed. This technique could contribute to standardization of LAPR.

\section{Conflict of interest}

The authors declare no conflict of interest.

\section{References}

1. den Dulk M, Marijnen CA, Putter H, et al. Risk factors for adverse outcome in patients with rectal cancer treated with an abdominoperineal resection in the total mesorectal excision trial. Ann Surg 2007; 246: 83-90.

2. den Dulk M, Putter $H$, Collette L, et al. The abdominoperineal resection itself is associated with an adverse outcome: the European experience based on a pooled analysis of five European randomised clinical trials on rectal cancer. Eur J Cancer 2009; 45: $1175-83$

3. Marr R, Birbeck K, Garvican J, et al. The modern abdominoperineal excision: the next challenge after total mesorectal excision. Ann Surg 2005; 242: 74-82.

4. Wibe A, Syse A, Andersen E, et al. Oncological outcomes after total mesorectal excision for cure for cancer of the lower rectum: anterior vs. abdominoperineal resection. Dis Colon Rectum 2004; 47: 48-58.

5. Holm T, Ljung A, Haggmark T, et al. Extended abdominoperineal resection with gluteus maximus flap reconstruction of the pelvic floor for rectal cancer. Br J Surg 2007; 94: 232-8.

6. West NP, Anderin C, Smith KJ, et al. European Extralevator Abdominoperineal Excision Study G: multicentre experience with extralevator abdominoperineal excision for low rectal cancer. Br J Surg 2010; 97: 588-99. 
7. West NP, Finan PJ, Anderin C, et al. Evidence of the oncologic superiority of cylindrical abdominoperineal excision for low rectal cancer. J Clin Oncol 2008; 26: 3517-22.

8. Prytz M, Angenete E, Ekelund J, et al. Extralevator abdominoperineal excision (ELAPE) for rectal cancer--short-term results from the Swedish Colorectal Cancer Registry. Selective use of ELAPE warranted. Int J Colorectal Dis 2014; 29: 981-7.

9. Chi P, Chen ZF, Lin HM, et al. Laparoscopic extralevator abdominoperineal resection for rectal carcinoma with transabdominal levator transection. Ann Surg Oncol 2013; 20: 1560-6.

10. Han JG, Wang ZJ, Wei GH, et al. Randomized clinical trial of conventional versus cylindrical abdominoperineal resection for locally advanced lower rectal cancer. Am J Surg 2012; 204: 274-82.

11. Han JG, Wang ZJ, Qian Q, et al. A prospective multicenter clinical study of extralevator abdominoperineal resection for locally advanced low rectal cancer. Dis Colon Rectum 2014; 57: 1333-40.

12. Horch RE, D'Hoore A, Holm T, et al. Laparoscopic abdominoperineal resection with open posterior cylindrical excision and primary transpelvic VRAM flap. Ann Surg Oncol 2012; 19: 502-3.

13. Prytz M, Angenete E, Bock D, et al. Extralevator abdominoperineal excision for low rectal cancer-extensive surgery to be used with discretion based on 3-year local recurrence results: a Registry-based, Observational National Cohort Study. Ann Surg 2016; 263: 516-21.

Received: 18.05.2016, accepted: 4.07.2016. 